\title{
Reduced-Order $l_{2}-l_{\infty}$ Filter Design for a Class of Discrete-Time Nonlinear Systems with Multiple Sensor Faults
}

\author{
Wenbai $\mathrm{Li}^{1}$ and Huxiong $\mathrm{Li}^{2}$ \\ ${ }^{1}$ College of Physics and Electronic Information Engineering, Wenzhou University, Wenzhou, Zhejiang 325035, China \\ ${ }^{2}$ Oujiang College, Wenzhou University, Wenzhou, Zhejiang 325027, China \\ Correspondence should be addressed to Huxiong Li; huxiongli12@gmail.com
}

Received 8 March 2013; Revised 12 June 2013; Accepted 27 June 2013

Academic Editor: Bo Shen

Copyright (c) $2013 \mathrm{~W}$. Li and H. Li. This is an open access article distributed under the Creative Commons Attribution License, which permits unrestricted use, distribution, and reproduction in any medium, provided the original work is properly cited.

\begin{abstract}
The reduced-order filtering problem for a class of discrete-time smooth nonlinear systems subject to multiple sensor faults is studied. It is well known that a smooth complex nonlinear system can be approximated by a Takagi-Sugeno fuzzy linear system with finite number of subsystems. In this work, firstly, the discrete-time smooth nonlinear system is transferred into a Takagi-Sugeno fuzzy linear system with finite number of subsystems. Secondly, a filter with the reduced order of the original system is proposed to be designed. Different from the traditional assumption in which the measurement of the output is ideal, the measurement of the output is subject to sensor faults which are described via Bernoulli processes. By using the augmentation technique, a stochastic TakagiSugeno filtering error system is obtained. For the stochastic filtering error system, the exponential stability and the energy-to-peak performance are investigated. Sufficient conditions which can guarantee the exponential stability and the $l_{2}-l_{\infty}$ performance are obtained. Then, with the proposed conditions, the design procedure of the filter for the nonlinear system is proposed. Finally, a numerical example is used to show the effectiveness of the proposed design methodology.
\end{abstract}

\section{Introduction}

During the last decades, the robust filter design problem has been paid a lot of efforts due to the fact that there are a lot of practical applications. Among all the published filtering works, Kalman filtering has been shown the great effective to many systems since it was proposed in the 1960s. However, in the Kalman filter design, it requires the precise linear system model and the covariance of the measurement noise at each sampling time. In addition, the system may be not only subject to the measurement noise but also the external disturbances. Due to these facts, recently, there are many new works on the robust filtering which can attenuate the effect of the external disturbances and robust against the system uncertainties $[1,2]$.

By assuming that the external disturbance is $l_{2}$ bounded, there are energy-to-energy filtering [3-9], energy-to-peak filtering [10-16], and peak-to-peak filtering [17] in the new filtering aspects. In the energy-to-energy filter design, the noise is assumed to be arbitrary, but, with $L_{2}$-bounded energy, the objective is to minimize the upper bound of the $L_{2}$ gain from the external disturbances to the estimation error.
While in the peak-to-peak filter design, the infinity norm of the filtering error aims to be minimized when the infinity norm of the external disturbance is bounded; see [17] and the references therein. In the energy-to-peak filter design, the infinity norm of the filtering error is minimized when the energy of the disturbance is $l_{2}$ bounded. If the peak value of the filtering error is constrained to be within a certain range or required to be minimized, the energy-to-peak filtering strategy is one of the best strategies in the robust filtering. The energy-to-peak performance was originally studied in [18]. Since this strategy is effective in many applications, the energy-to-peak filtering has been applied to many other systems; see $[19,20]$.

On the other hand, the research on the smooth nonlinear system which can be approximated by Takagi-Sugeno fuzzy linear system with finite number of subsystems is a hot topic [21-43] since the techniques for the linear systems can be applied to the Takagi-Sugeno fuzzy systems. Therefore, unsurprisingly, the study of the fuzzy systems based on Takagi-Sugeno [44] model has attracted a lot of attention $[45,46]$. In [34], the filtering problem for discrete-time 


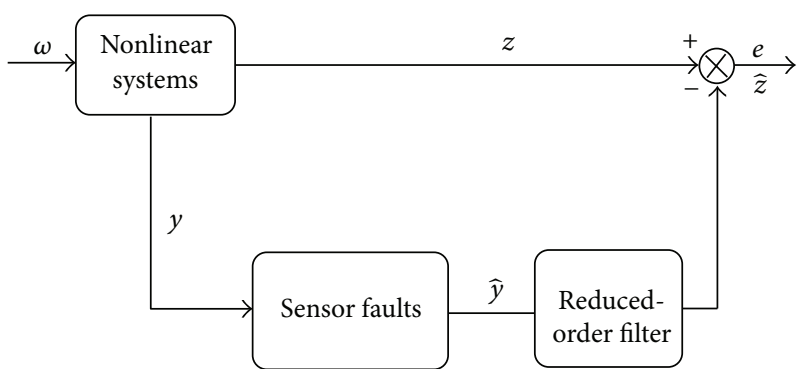

FIGURE 1: Filtering problem for nonlinear systems with sensor faults.

Takagi-Sugeno fuzzy systems with time-varying delays was studied. The parameters of the filter can be determined by solving the linear matrix inequalities. In $[40,47]$, the decentralized fuzzy filter design problem for nonlinear interconnected systems was studied.

In traditional filtering problem, it is always assumed that the measurements of the output are perfect; that is, the output of the system is available to the filter. However, in many cases, the measurements may not be available to the filter such as the sensors are subject to faults. If these faults were not considered during the filter design procedure, it is obvious that the existing faults would affect the filter. An extreme example is that the sensors do not work at all. The filter cannot use the measurement to update the estimation. Therefore, it is necessary to consider the sensor faults during the filter design. The filtering problem with the sensor faults has attracted a lot of attention recently [48-57]. In [56], the $H_{\infty}$ filtering for discrete-time stochastic systems with missing measurements was studied. In [54], the robust setmembership filtering for systems with missing measurement was studied via a linear matrix inequality approach. In [53], the variance-constrained $H_{\infty}$ filtering for a class of nonlinear time-varying systems with multiple missing measurements was investigated for finite-horizon case. Though there are many works on the filtering problem with sensor faults, in the literature, there are few results on the reduced-order energyto-peak filtering for discrete-time nonlinear systems with sensor faults.

In this paper, the reduced-order filtering problem for a class of discrete-time smooth nonlinear systems with multiple sensor faults is exploited. It is well known that a smooth complex nonlinear system can be approximated by a Takagi-Sugeno fuzzy linear system with finite number of subsystems. In the design, the smooth nonlinear system is firstly transferred into a Takagi-Sugeno fuzzy linear system with finite number of subsystems. Then, a reduced-order filter which is also dependent on the normalized fuzzy-weighting function is proposed to be designed. By defining the filtering error, with the augmentation skill and consideration of the sensor faults, a stochastic Takagi-Sugeno fuzzy filtering error system is obtained. Studying the exponential stability and the energy-to-peak performance of the filtering error system, the design method for the filter is proposed. Finally, a numerical example is used to show the effectiveness of the proposed design methodology. The contribution of this paper can be summarized as follows. (1) The sensor faults are considered in the energy-to-peak filtering problem, which makes the work more generalized. (2) The filter is a reduced-order filter, which makes the results more attractive in the design for high-order and complex systems.

\section{Problem Formulation}

In this work, a class of smooth discrete-time nonlinear systems which can be modeled by the following T-S fuzzy linear models is considered as follows.

Plant Rule i. IF $\eta_{1}(k)$ is $\mathcal{N}_{i 1}$ and $\eta_{2}(k)$ is $\mathscr{N}_{i 2}, \ldots$ and $\eta_{s}(k)$ is $\mathcal{N}_{i s}$, THEN

$$
\begin{gathered}
x_{k+1}=A_{i} x_{k}+B_{i} \omega_{k}, \\
y_{k}=C_{i} x_{k}+D_{i} \omega_{k}, \\
z_{k}=G_{i} x_{k},
\end{gathered}
$$

where $\mathcal{N}_{i j}$ for $i=1, \ldots, s_{1}, j=1, \ldots, s$ are the fuzzy sets, $\eta_{k}=\left[\eta_{1 k}, \eta_{2 k}, \ldots, \eta_{s k}\right]$ is the premise variable vector, $s$ denotes the number of the premise variable, $s_{1}$ represents the number of fuzzy rule, $x_{k} \in \mathbb{R}^{n}$ is the state vector, $\omega_{k}$ is the external disturbance, $y_{k} \in \mathbb{R}^{p}$ is the systems output which is measured by corresponding sensors, $z_{k}$ stands for the signal to be filtered, and $A_{i}, B_{i}, C_{i}, D_{i}$, and $G_{i}$ are given constant matrices.

In order to deal with the fuzzy rules, the normalized fuzzy-membership functions are defined as follows:

$$
\mu_{i}(\eta(k))=\frac{\prod_{j=1}^{s} \theta_{i j}\left(\eta_{j}(k)\right)}{\sum_{i=1}^{s_{1}} \prod_{j=1}^{s} \theta_{i j}\left(\eta_{j}(k)\right)} .
$$

Here, $\theta_{i j}\left(\eta_{j}(k)\right)$ stands for the grade of the membership of $\eta_{j}(k)$ in the $i$ th fuzzy rule. It is noted that the normalized fuzzy-membership functions have the following properties:

$$
\begin{gathered}
0 \leq \mu_{i}(\eta(k)) \leq 1, \\
\sum_{i=1}^{s_{1}} \mu_{i}(\eta(k))=1 .
\end{gathered}
$$

The filter problem considered in this paper is shown in Figure 1. The nonlinear system is subject to an external disturbance $\omega_{k}$. The output of the systems is $y_{k}$ which is subject to sensor faults and the measurement of the output is described with

$$
\widehat{y}_{k}=\Xi_{k} \sum_{i=1}^{s_{1}} \mu_{i}(\eta(k)) C_{i} x_{k}+D \omega_{k}
$$

where $\Xi_{k}=\operatorname{diag}\left\{\alpha_{1, k}, \ldots, \alpha_{o, k}, \ldots, \alpha_{p, k}\right\}$, and $\alpha_{o, k}$ is a Bernoulli process with the probability of $\alpha_{o, k}=1$ is $\beta_{o}, o=$ $1, \ldots, p, \widehat{z}_{k}$ is the output of the filter; that is, $\widehat{z}_{k}$ is the estimation of $z_{k}$.

In the reduce-order filter design for T-S fuzzy systems, the filter has the following structure.

Filter Rule i. IF $\theta_{1}(k)$ is $\mathcal{N}_{i 1}, \theta_{2}(k)$ is $\mathcal{N}_{i 2}, \ldots$, and $\theta_{s}(k)$ is $\mathcal{N}_{i s}$, THEN

$$
\begin{gathered}
\widehat{x}_{k+1}=A_{f i} \widehat{x}_{k}+B_{f i} \widehat{y}_{k}, \\
\widehat{z}_{k}=G_{f i} \widehat{x}_{k}, \\
i=1, \ldots, s_{1},
\end{gathered}
$$


where $\widehat{x}_{k} \in \mathbb{R}^{n_{1}}\left(n_{1}<n\right)$ is the state in the filter, $\widehat{z}_{k}$ is the output of the filter, and $A_{f i}, B_{f i}, G_{f i}$ are filter parameters to be determined.

Defining a filtering error as $e_{k}:=z_{k}-\widehat{z}_{k}$, the filtering error system is as follows:

$$
\begin{gathered}
\xi_{k+1}=\bar{A}(\eta(k)) \xi_{k}+\bar{A}_{m}(\eta(k)) \xi_{k}+\bar{B}(\eta(k)) \omega_{k}, \\
e_{k}=\bar{G}(\eta(k)) \xi_{k} .
\end{gathered}
$$

Here, the following notations are defined:

$$
\begin{gathered}
\xi_{k}=\left[\begin{array}{l}
x_{k} \\
\hat{x}_{k}
\end{array}\right], \quad \bar{\Xi}=\operatorname{diag}\left\{\beta_{1}, \ldots, \beta_{p}\right\}, \\
\bar{A}(\eta(k))=\left[\begin{array}{cc}
A(\eta(k)) & 0 \\
B_{f}(\eta(k)) \bar{\Xi} C(\eta(k)) & A_{f}(\eta(k))
\end{array}\right], \\
\bar{A}_{m}(\eta(k))=\left[\begin{array}{cc}
0 & 0 \\
B_{f}(\eta(k))\left(\Xi_{k}-\bar{\Xi}\right) C(\eta(k)) & 0
\end{array}\right], \\
\bar{B}(\eta(k))=\left[\begin{array}{c}
B(\eta(k)) \\
B_{f}(\eta(k)) D(\eta(k))
\end{array}\right], \\
\bar{G}(\eta(k))=\left[G(\eta(k))-G_{f}(\eta(k))\right] .
\end{gathered}
$$

Since the system parameters are dependent on parameters of each subsystem and are time-varying, we use the following notations to represents these parameters:

$$
\begin{aligned}
& A(\eta(k))=\sum_{i=1}^{s_{1}} \mu_{i}(\eta(k)) A_{i}, \\
& B(\eta(k))=\sum_{i=1}^{s_{1}} \mu_{i}(\eta(k)) B_{i}, \\
& C(\eta(k))=\sum_{i=1}^{s_{1}} \mu_{i}(\eta(k)) C_{i}, \\
& D(\eta(k))=\sum_{i=1}^{s_{1}} \mu_{i}(\eta(k)) D_{i}, \\
& G(\eta(k))=\sum_{i=1}^{s_{1}} \mu_{i}(\eta(k)) G_{i},
\end{aligned}
$$

$$
\begin{aligned}
A_{f}(\eta(k)) & =\sum_{i=1}^{s_{1}} \mu_{i}(\eta(k)) A_{f i}, \\
B_{f}(\eta(k)) & =\sum_{i=1}^{s_{1}} \mu_{i}(\eta(k)) B_{f i}, \\
G_{f}(\eta(k)) & =\sum_{i=1}^{s_{1}} \mu_{i}(\eta(k)) G_{f i} .
\end{aligned}
$$

In the filtering problem, the main objective is to minimize the filtering error. Since the external disturbance is unknown, the energy-to-peak gain $\gamma$ is introduced to evaluate the effect from the external input to the filtering error: for zero-initial condition, the energy-to-peak gain from the external input $\omega_{k}$ to the filtering error $e_{k}$ should be smaller than the index $\gamma$; that is,

$$
\mathscr{E}\left\{\|e\|_{\infty}\right\}<\gamma\|\omega\|_{2},
$$

where $\mathscr{E}\left\{\|e\|_{\infty}\right\}$ is the expectation of the $\infty$-norm of the filtering error $e_{k}$ and $\|\omega\|_{2}$ indicates the 2-norm of the external input $\omega_{k}$.

In summary, the design objectives of the paper are as follows.

(1) The filtering error system in (6) is exponentially stable for zero disturbance input $\omega_{k}$.

(2) For zero-initial condition, the energy-to-peak gain from the external input to the filtering error should be lower than a prescribed level $\gamma$.

\section{Main Results}

In this section, by assuming that the parameters for each subfilter are given, the exponentially stability and energy-topeak performance of the filtering error system will be studied. Based on the analysis results, the filter design method will be proposed.

3.1. Exponential Stability and Energy-to-Peak Performance Analysis. The following theorem provides the condition for the exponential stability of the filtering error system.

Theorem 1. Consider a discrete-time nonlinear system in (1) and suppose that $A_{f}(\eta(k)), B_{f}(\eta(k))$, and $G_{f}(\eta(k))$ are given. The filtering error system in (6) is exponentially stable if there exist matrices $P(\eta(k))=P^{T}(\eta(k))>0$ and $P(\eta(k+1))=$ $P^{T}(\eta(k+1))>0$ such that

$$
\left.\begin{array}{c}
\sqrt{\beta_{1}\left(1-\beta_{1}\right)} P(\eta(k+1)) \bar{A}_{1, m}(\eta(k)) \\
\vdots \\
\sqrt{\beta_{p}\left(1-\beta_{p}\right)} P(\eta(k+1)) \bar{A}_{p, m}(\eta(k)) \\
P(\eta(k+1)) \bar{A}(\eta(k)) \\
-P(\eta(k))
\end{array}\right]<0,
$$


where

$$
\begin{array}{r}
\bar{A}_{o, m}=\left[\begin{array}{cc}
0 & 0 \\
B_{f}(\eta(k)) \Theta_{o} C(\eta(k)) & 0
\end{array}\right], \\
\Theta_{o}=\operatorname{diag}\{0, \ldots, 1 \ldots, 0\} .
\end{array}
$$

Proof. Choose a Lyapunov function candidate for the filtering error system in (6) as follows:

$$
V\left(\xi_{k}, \eta(k), k\right)=\xi_{k}^{T} P(\eta(k)) \xi_{k}
$$

where $P(\eta(k))=P^{T}(\eta(k))>0$ is a Lyapunov matrix which is also dependent on the normalized fuzzy-weighting functions. Since there is a stochastic variable $\alpha_{o, k}$ in the filtering error system, the difference of the Lyapunov function is defined as

$$
\begin{aligned}
\Delta V & \left(\xi_{k}, \eta(k), k\right) \\
= & \mathscr{E}\left\{V\left(\xi_{k+1}, \eta(k+1), k+1\right) \mid V\left(\xi_{k}, \eta(k), k\right)\right\} \\
& -V\left(\xi_{k}, \eta(k), k\right) .
\end{aligned}
$$

Considering the dynamics of the unforced filtering error system, the difference of the Lyapunov function is equal to
$\Delta V\left(\xi_{k}, \eta(k), k\right)$

$$
\begin{aligned}
=\xi_{k}^{T}\{ & \bar{A}^{T}(\eta(k)) P(\eta(k+1)) \bar{A}(\eta(k)) \\
& \times \sum_{o=1}^{p} \beta_{o}\left(1-\beta_{o}\right) \bar{A}_{o, m}^{T}(\eta(k)) P(\eta(k+1)) \\
& \left.\times \bar{A}_{o, m}(\eta(k))-P(\eta(\mathrm{k}))\right\} \xi_{k} .
\end{aligned}
$$

In terms of Schur complement, the condition in (10) can assure that the difference of the Lyapunov function $\Delta V\left(\xi_{k}, \eta(k), k\right)$ is negative. Thus, the filtering error system is exponentially stable. The proof is completed.

In Theorem 1, by assuming the external disturbance is zero, the exponential stability is studied. In the following, the effect of the external disturbance will be evaluated.

Theorem 2. Consider a discrete-time nonlinear system in (1) and suppose that $A_{f}(\eta(k)), B_{f}(\eta(k)), G_{f}(\eta(k))$, and $a$ positive scalar $\gamma$ are given. The filtering error system in (6) is exponentially stable and the energy-to-peak performance is guaranteed if there exist matrices $P(\eta(k))=P^{T}(\eta(k))>0$ and $P(\eta(k+1))=P^{T}(\eta(k+1))>0$ such that

$$
\left[\begin{array}{cccccc}
-P(\eta(k+1)) & \cdots & 0 & 0 & \sqrt{\beta_{1}\left(1-\beta_{1}\right)} P(\eta(k+1)) \bar{A}_{1, m}(\eta(k)) & 0 \\
* & \ddots & \vdots & \vdots & \vdots & \vdots \\
* & * & -P(\eta(k+1)) & 0 & \sqrt{\beta_{p}\left(1-\beta_{p}\right)} P(\eta(k+1)) \bar{A}_{p, m}(\eta(k)) & 0 \\
* & * & * & -P(\eta(k+1)) & P(\eta(k+1)) \bar{A}(\eta(k)) & P(\eta(k+1)) \bar{B}(\eta(k)) \\
* & * & * & * & -P(\eta(k)) & 0 \\
* & * & * & * & *
\end{array}\right]<0,
$$

$$
\left[\begin{array}{cc}
P(\eta(k)) & \bar{G}^{T}(\eta(k)) \\
* & \gamma^{2} I
\end{array}\right]>0
$$

Proof. According to Schur complement, the condition in (15) can guarantee that

$$
\begin{gathered}
\mathscr{E}\left\{V\left(\xi_{k+1}, \eta(k+1), k+1\right) \mid V\left(\xi_{k}, \eta(k), k\right)\right\} \\
-V\left(\xi_{k}, \eta(k), k\right)-\omega_{k}^{T} \omega_{k}<0 .
\end{gathered}
$$

When the external disturbance is zero, that is, $\omega_{k}=0$, inequality (17) is reduced to

$$
\begin{aligned}
\mathscr{E}\{V & \left.\left(\xi_{k+1}, \eta(k+1), k+1\right) \mid V\left(\xi_{k}, \eta(k), k\right)\right\} \\
& -V\left(\xi_{k}, \eta(k), k\right)<0 .
\end{aligned}
$$

According to Theorem 1, the filtering error system is exponentially stable.

Now we are going to study the energy-to-peak performance attenuation level $\gamma$. Considering the nonzero external input, it infers from (18) that the difference of the Lyapunov function satisfies

$$
\Delta V\left(\xi_{k}, \eta(k), k\right)<\omega_{k}^{T} \omega_{k} .
$$

Suppose that the initial states of the system and the filter are both zeros. Then, the Lyapunov function satisfies

$$
V\left(\xi_{k}, \eta(k), k\right)=\sum_{i=0}^{k-1} \Delta V<\sum_{i=0}^{k-1} \omega_{i}^{T} \omega_{i}
$$

By using Schur complement, the inequality in (16) is equivalent to

$$
\bar{G}^{T}(\eta(k)) \bar{G}(\eta(k))<\gamma^{2} P(\eta(k)) .
$$


Recalling the dynamics of the filtering error system (6), (12), and (20), one gets

$$
\begin{aligned}
\mathscr{E}\left\{e_{k}^{T} e_{k}\right\} & =\left[\xi_{k}\right]^{T} \bar{G}^{T}(\eta(k)) \bar{G}(\eta(k))\left[\xi_{k}\right] \\
& <\gamma^{2}\left[\xi_{k}\right]^{T} P(\eta(k))\left[\xi_{k}\right] \\
& =\gamma^{2}\left(V\left(\xi_{k}, \eta(k), k\right)+\omega_{k}^{T} \omega_{k}\right)<\gamma^{2} \sum_{i=0}^{\infty} \omega_{i}^{T} \omega_{i} .
\end{aligned}
$$

Taking the supremum over time $k>0$ to the previous inequality results to the following condition $\mathscr{E}\left\{\|e\|_{\infty}\right\}<$ $\gamma\|\omega\|_{2}$ for all nonzero $\omega_{k} \in l_{2}[0, \infty)$. Therefore, the second objective is satisfied; that is, conditions (15) and (16) can guarantee the exponential stability and the energy-to-peak attenuation level $\gamma$. The proof is completed.

In Theorem 2, the energy-to-peak performance has been investigated. However, the obtained conditions are only sufficient. In order to derive less conservative results, the following theorem is introduced.

Theorem 3. Consider a discrete-time nonlinear system in (1) and suppose that $A_{f}(\eta(k)), B_{f}(\eta(k)), G_{f}(\eta(k))$, and a positive scalar $\gamma$ are given. The filtering error system in (6) is exponentially stable and the energy-to-peak performance is guaranteed if there exist matrices $M(\eta(k)), P(\eta(k))=$ $P^{T}(\eta(k))>0$, and $P(\eta(k+1))=P^{T}(\eta(k+1))>$ such that

$$
\begin{gathered}
{\left[\begin{array}{cccccc}
\Omega_{1} & \cdots & 0 & 0 & \Omega_{1,2} & 0 \\
* & \ddots & \vdots & \vdots & \vdots & \vdots \\
* & * & \Omega_{1} & 0 & \Omega_{p, 2} & 0 \\
* & * & * & \Omega_{1} & M(\eta(k)) \bar{A}(\eta(k)) & M(\eta(k)) \bar{B}(\eta(k)) \\
* & * & * & * & -P(\eta(k)) & 0 \\
* & * & * & * & * & -I
\end{array}\right]} \\
\\
<0, \\
\end{gathered}
$$

where

$$
\begin{gathered}
\Omega_{1}=P(\eta(k+1))-M(\eta(k))-M^{T}(\eta(k)), \\
\Omega_{o, 2}=\sqrt{\beta_{o}\left(1-\beta_{o}\right)} M(\eta(k)) \bar{A}_{o, m}(\eta(k)) .
\end{gathered}
$$

Proof. By choosing $M(\eta(k))=M^{T}(\eta(k))=P(\eta(k+1))$, the condition in (23) is reduced to the condition in (15). On the other hand, with the fact that $(M(\eta(k))-P(\eta(k+1))) P^{-1}(\eta(k+$ 1)) $(M(\eta(k))-P(\eta(k+1)))^{T} \geq 0$, which implies that $P(\eta(k+$
1)) $-M(\eta(k))-M^{T}(\eta(k)) \geq-M(\eta(k)) P^{-1}(\eta(k+1)) M^{T}(\eta(k))$, one gets

$$
\left[\begin{array}{cccccc}
\bar{\Omega}_{1} & \cdots & 0 & 0 & \Omega_{1,2} & 0 \\
* & \ddots & \vdots & \vdots & \vdots & \vdots \\
* & * & \bar{\Omega}_{1} & 0 & \Omega_{p, 2} & 0 \\
* & * & * & \bar{\Omega}_{1} & M(\eta(k)) \bar{A}(\eta(k)) & M(\eta(k)) \bar{B}(\eta(k)) \\
* & * & * & * & -P(\eta(k)) & 0 \\
* & * & * & * & * & -I \\
& & & & &
\end{array}\right.
$$

where

$$
\bar{\Omega}_{1}=-M(\eta(k)) P^{-1}(\eta(k+1)) M^{T}(\eta(k)) .
$$

By performing a congruence transformation, Theorem 3 is equivalent to Theorem 2 .

3.2. Filter Design Approach. Based on the results in Theorem 3, the filter design method will be developed in this subsection

Theorem 4. Consider a discrete-time nonlinear system in (1) and give a positive scalar $\gamma$. Then, the filtering error system in (6) is exponentially stable with a reduced-order filter in the form of (5) if there exist matrices $\left[\begin{array}{cc}P_{11, i} & P_{12, i} \\ * & P_{22, i}\end{array}\right]=\left[\begin{array}{cc}P_{11, i} & P_{12, i} \\ * & P_{22, i}\end{array}\right]^{T}>0$, [1pt] $\left[\begin{array}{cc}M_{11, i} & N M_{12} \\ M_{21, i} & M_{12}\end{array}\right], A F_{i}, B F_{i}$, and $G F_{i}$, for all $i=1, \ldots, s_{1}, j=$ $i, \ldots, s_{1}, r=1, \ldots, s_{1}$, such that the following conditions hold

$$
\begin{gathered}
{\left[\begin{array}{cc}
\Omega_{15} & \Omega_{16} \\
* & \gamma^{2} I
\end{array}\right]>0,} \\
\mathscr{H}_{i, j, r}+\mathscr{H}_{j, i, r}<0,
\end{gathered}
$$

where

$$
\begin{aligned}
& \mathscr{H}_{i, j, r}=\left[\begin{array}{cccccc}
\Omega_{11} & \cdots & 0 & 0 & \Omega_{1,12} & 0 \\
* & \ddots & \vdots & \vdots & \vdots & \vdots \\
* & * & \Omega_{11} & 0 & \Omega_{p, 12} & 0 \\
* & * & * & \Omega_{11} & \Omega_{13} & \Omega_{14} \\
* & * & * & * & -\Omega_{15} & 0 \\
* & * & * & * & * & -I
\end{array}\right], \\
& \Omega_{11}=\left[\begin{array}{cc}
P_{11, r} & P_{12, r} \\
* & P_{22, r}
\end{array}\right]-\left[\begin{array}{cc}
M_{11, i} & N M_{12} \\
M_{21, i} & M_{12}
\end{array}\right]-\left[\begin{array}{cc}
M_{11, i} & N M_{12} \\
M_{21, i} & M_{12}
\end{array}\right]^{T} \text {, } \\
& \Omega_{o, 12}=\sqrt{\beta_{o}\left(1-\beta_{o}\right)}\left[\begin{array}{cc}
N B F_{i} \Theta_{o} C_{j} & 0 \\
B F_{i} \Theta_{o} C_{j} & 0
\end{array}\right], \\
& \Omega_{13}=\left[\begin{array}{cc}
M_{11, i} A_{j}+N B F_{i} \bar{\Xi} C_{j} & N A F_{i} \\
M_{21, i} A_{j}+B F_{i} \bar{\Xi} C_{j} & A F_{i}
\end{array}\right] \text {, } \\
& \Omega_{14}=\left[\begin{array}{c}
M_{11, i} B_{j}+N B F_{i} D_{j} \\
M_{21, i} B_{j}+B F_{i} D_{j}
\end{array}\right], \\
& \Omega_{15}=\left[\begin{array}{cc}
P_{11, i} & P_{12, i} \\
* & P_{22, i}
\end{array}\right] \text {, }
\end{aligned}
$$




$$
\begin{gathered}
\Omega_{16}=\left[\begin{array}{c}
G_{i}^{T} \\
-G F_{i}^{T}
\end{array}\right], \\
N=\left[\begin{array}{l}
I \\
0
\end{array}\right] .
\end{gathered}
$$

Moreover, the parameters for each subfilter can be determined by the following equations:

$$
A_{f i}=M_{12}^{-1} A F_{i}, \quad B_{f i}=M_{12}^{-1} B F_{i}, \quad G_{f i}=G F_{i} .
$$

Proof. The proof of the theorem can be done by partitioning these matrices in Theorem 3 as

$$
P_{i}=\left[\begin{array}{cc}
P_{11, i} & P_{12, i} \\
* & P_{22, i}
\end{array}\right], \quad M_{i}=\left[\begin{array}{cc}
M_{11, i} & N M_{12} \\
M_{21, i} & M_{12}
\end{array}\right] .
$$

This completes the proof.

It is noted that the filter error can be smaller if the performance level $\gamma$ is smaller when the system is subject to the same disturbance. So it is meaningful to optimize the value for $\gamma$. The minimum value for $\gamma$ can be derived by the following corollary.

Corollary 5. The minimum energy-to-peak performance index $\gamma$ for the filtering error system in (6) can be found by solving the following convex optimization problem:

$$
\begin{array}{ll}
\min & \gamma^{2}, \\
\text { s.t. } & (28), \\
& \forall i \leq j, i, r, j=1, \ldots, s_{1} .
\end{array}
$$

3.3. Comparison with Robust $H_{\infty}$ Filter. In order to compare with the $H_{\infty}$ filtering, we are going to study the $H_{\infty}$ performance of the filtering error system and propose the robust $H_{\infty}$ filter. The $H_{\infty}$ performance index is defined as follows:

$$
\mathscr{E}\left\{\|e\|_{2}\right\}<\gamma\|\omega\|_{2},
$$

where $\mathscr{E}\left\{\|e\|_{2}\right\}$ is the expectation of the 2 -norm of the filtering error $e_{k}$ and $\|\omega\|_{2}$ indicates the 2 -norm of the external input $\omega_{k}$.

Theorem 6. Consider a discrete-time nonlinear system in (1) and suppose that $A_{f}(\eta(k)), B_{f}(\eta(k)), G_{f}(\eta(k))$, and a positive scalar $\gamma$ are given. The filtering error system in (6) is exponentially stable and the $H_{\infty}$ performance is guaranteed if there exist matrices $M(\eta(k)), P(\eta(k))=P^{T}(\eta(k))>0$, and $P(\eta(k+1))=P^{T}(\eta(k+1))>0$ such that

$$
\left[\begin{array}{ccccccc}
\Omega_{1} & \cdots & 0 & 0 & 0 & \Omega_{1,2} & 0 \\
* & \ddots & \vdots & \vdots & \vdots & \vdots & \vdots \\
* & * & \Omega_{1} & 0 & 0 & \Omega_{p, 2} & 0 \\
* & * & * & \Omega_{1} & 0 & M(\eta(k)) \bar{A}(\eta(k)) & M(\eta(k)) \bar{B}(\eta(k)) \\
* & * & * & * & -I & \bar{G}(\eta(k)) & 0 \\
* & * & * & * & * & -P(\eta(k)) & 0 \\
* & * & * & * & * & * & -\gamma^{2} I
\end{array}\right]
$$

$<0$.
Proof. The proof can be done as similar lines in $[58,59]$ and the proof in the previous subsections.

Similarly, we have the following filter design method.

Theorem 7. Consider a discrete-time nonlinear system in (1) and give a positive scalar $\gamma$. Then, the filtering error system in (6) is exponentially stable with a reduced-order filter in the form of (5) and the $H_{\infty}$ performance is satisfied if there exist matrices $\left[\begin{array}{cc}P_{11, i} & P_{12, i} \\ * & P_{22, i}\end{array}\right]=\left[\begin{array}{cc}P_{11, i} & P_{12, i} \\ * & P_{22, i}\end{array}\right]^{T}>0,\left[\begin{array}{cc}M_{11, i} & N M_{12} \\ M_{21, i} & M_{12}\end{array}\right], A F_{i}, B F_{i}$, and $G F_{i}$, for all $i=1, \ldots, s_{1}, j=i, \ldots, s_{1}, r=1, \ldots, s_{1}$, such that the following conditions hold:

$$
\mathscr{H}_{i, j, r}+\mathscr{H}_{j, i, r}<0
$$

where

$$
\mathscr{H}_{i, j, r}=\left[\begin{array}{ccccccc}
\Omega_{11} & \cdots & 0 & 0 & 0 & \Omega_{1,12} & 0 \\
* & \ddots & \vdots & \vdots & \vdots & \vdots & \vdots \\
* & * & \Omega_{11} & 0 & 0 & \Omega_{p, 12} & 0 \\
* & * & * & \Omega_{11} & 0 & \Omega_{13} & \Omega_{14} \\
* & * & * & * & -I & {\left[G-G F_{i}\right]} & 0 \\
* & * & * & * & * & -\Omega_{15} & 0 \\
* & * & * & * & * & * & -\gamma^{2} I
\end{array}\right] .
$$

Moreover, the parameters for each subfilter can be determined by the following equations:

$$
A_{f i}=M_{12}^{-1} A F_{i}, \quad B_{f i}=M_{12}^{-1} B F_{i}, \quad G_{f i}=G F_{i} .
$$

Corollary 8. The minimum $H_{\infty}$ performance index $\gamma$ for the filtering error system in (6) can be found by solving the following convex optimization problem:

$$
\begin{array}{ll}
\min & \gamma^{2}, \\
\text { s.t. } & (35) \\
& \forall i \leq j, i, r, j=1, \ldots, s_{1} .
\end{array}
$$

\section{Illustrative Example}

In this section, a numeric example is given to show the design procedure of the proposed design method.

Consider a discrete-time smooth nonlinear system which is approximated by the following discrete-time T-S fuzzy system with two subsystems.

$$
\begin{aligned}
& \text { Plant Rule 1. IF } x_{1 k} \text { is } \mathcal{N}_{11} \text {, THEN } \\
& \qquad \begin{array}{c}
x_{k+1}=\left[\begin{array}{cc}
1 & 0.3 \\
-0.4 & 0.1
\end{array}\right] x_{k}+\left[\begin{array}{c}
-0.1 \\
-0.05
\end{array}\right] \omega_{k}, \\
y_{k}=\left[\begin{array}{ll}
1 & 0
\end{array}\right] x_{k}+0.01 \omega_{k}, \\
z_{k}=\left[\begin{array}{ll}
0 & 1
\end{array}\right] x_{k},
\end{array}
\end{aligned}
$$

Plant Rule 2. IF $x_{1 k}$ is $\mathcal{N}_{21}$, THEN

$$
\begin{gathered}
x_{k+1}=\left[\begin{array}{cc}
0.5 & -0.3 \\
-0.3 & 0.2
\end{array}\right] x_{k}+\left[\begin{array}{c}
0.1 \\
-0.1
\end{array}\right] \omega_{k}, \\
y_{k}=\left[\begin{array}{ll}
1 & 0
\end{array}\right] x_{k}+0.01 \omega_{k}, \\
z_{k}=\left[\begin{array}{ll}
0 & 1
\end{array}\right] x_{k},
\end{gathered}
$$


where $x_{1 k}$ is the first state of the system,

$$
\begin{aligned}
& \mathcal{N}_{11}= \begin{cases}0.5+\left|\frac{\sin \left(x_{1 k}\right)}{x_{1 k}}\right|, & \text { for } x_{1 k} \neq 0, \\
1, & \text { for } x_{1 k}=0,\end{cases} \\
& \mathcal{N}_{21}= \begin{cases}0.5-\left|\frac{\sin \left(x_{1 k}\right)}{x_{1 k}}\right|, & \text { for } x_{1 k} \neq 0, \\
0, & \text { for } x_{1 k}=0 .\end{cases}
\end{aligned}
$$

Considering the sensor fault, the probability of the available measurement is assumed to be 0.5. Since the order of the previous discrete-time nonlinear system is two, the order of the reduced-order filter is supposed to be one. By using Corollary 5, the obtained minimal energy-to-peak $\gamma$ is 0.1193 . The corresponding parameters of the filters are

$$
\begin{aligned}
& A_{f 1}=0.7386, \quad B_{f 1}=-0.5355, \quad G_{f 1}=0.3493, \\
& A_{f 2}=0.6507, \quad B_{f 2}=-0.6401, \quad G_{f 2}=0.3494 \text {. }
\end{aligned}
$$

By using Corollary 8 , the obtained minimal energy-to-peak $\gamma$ is 0.1647 . The corresponding parameters of the filters are

$$
\begin{array}{lll}
A_{f 1}=0.7102, & B_{f 1}=-0.4409, & G_{f 1}=0.4087, \\
A_{f 2}=0.4229, & B_{f 2}=-0.8466, & G_{f 2}=0.5215 .
\end{array}
$$

In order to verify the performance of the designed filter and compare it with the $H_{\infty}$ filtering, it is assumed that the initial state of the system is $[0.5,0.5]^{T}$. Figure 2 shows the filtering errors with different filters when the sensor is subject to the faults described in Figure 3. It can be seen that the reduced-order filter can track the signal well though the sensor is subject to faults. Moreover, the filtering error of the designed energy-to-peak filter is smaller than the one of $H_{\infty}$ filter. The 2-norm of the filtering error with the energy-topeak filter is 0.7246 . But the 2-norm of the filtering error with the $H_{\infty}$ filter is 0.8420 . The percentage of improvement is $13.94 \%$.

\section{Conclusions}

In this paper, the reduced-order energy-to-peak filter design problem for a class of discrete-time smooth nonlinear systems which can be modeled as T-S fuzzy linear systems was studied. The filter with the reduced order of the plant is dependent on the normalized fuzzy-weighting functions. The measurements of the output are subject to sensor faults which are described via a Bernoulli process. The exponential stability and the energy-to-peak performance for the filtering error system were studied. Based on the results, the filter design method was proposed. The parameters of the fuzzy filters can be obtained by solving a set of linear matrix inequalities. In the simulation section, a numeric example was used to show the procedure of the proposed design method. From the simulation results, the performance of the design filter is good.

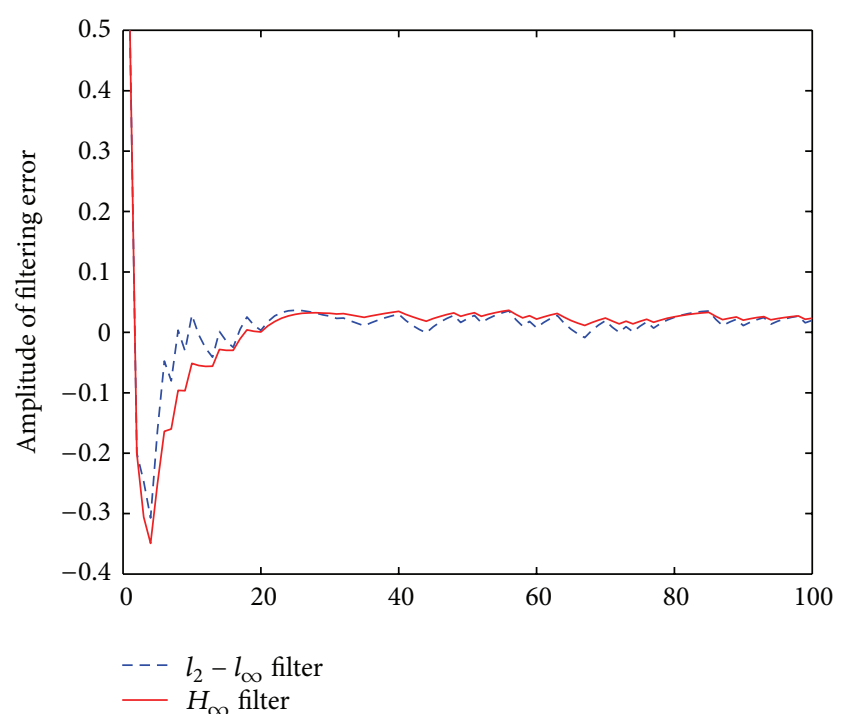

Figure 2: Signal to be estimated and the estimation.

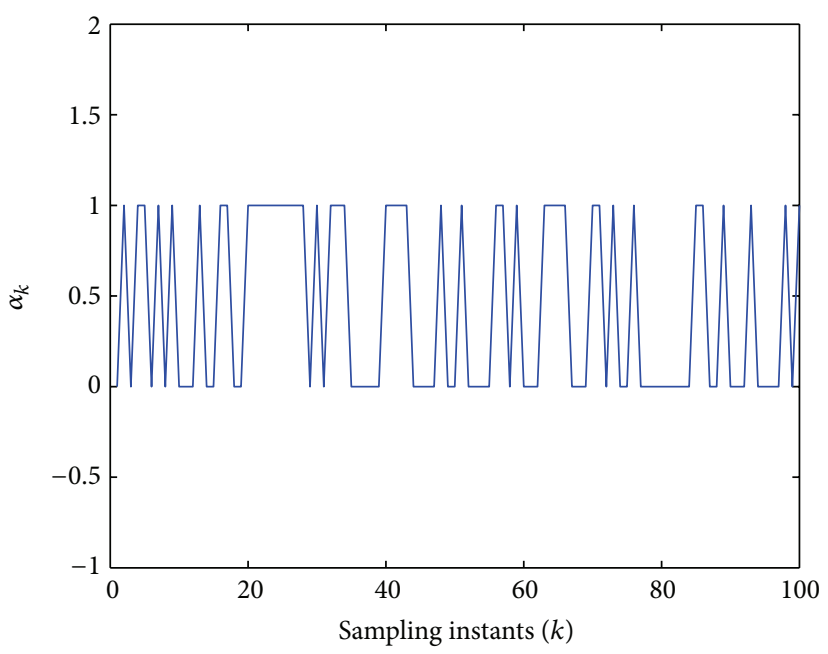

FIGURE 3: Stochastic sensor faults.

\section{Acknowledgments}

This work is supported by International Cooperation Projects of Zhejiang Province (no. 2012C24019) and Natural Science Foundation of Zhejiang Province (no. Y1100769).

\section{References}

[1] J. Xiong and J. Lam, "Fixed-order robust $H_{\infty}$ filter design for Markovian jump systems with uncertain switching probabilities," IEEE Transactions on Signal Processing, vol. 54, no. 4, pp. 1421-1430, 2006.

[2] S. Xu, J. Lam, T. Chen, and Y. Zou, "A delay-dependent approach to robust $H_{\infty}$ filtering for uncertain distributed delay systems," IEEE Transactions on Signal Processing, vol. 53, no. 10, part 1, pp. 3764-3772, 2005.

[3] W.-H. Chen and B.-S. Chen, "Robust filter for linear stochastic partial differential systems via a set of sensor measurements," 
IEEE Transactions on Circuits and Systems I, vol. 59, no. 6, pp. 1251-1264, 2012.

[4] Y. Xiao, Y.-Y. Cao, and Z. Lin, "Robust filtering for discrete-time systems with saturation and its application to transmultiplexers," IEEE Transactions on Signal Processing, vol. 52, no. 5, pp. 1266-1277, 2004.

[5] C. E. de Souza, A. Trofino, and K. A. Barbosa, "Modeindependent $H_{\infty}$ filters for Markovian jump linear systems," IEEE Transactions on Automatic Control, vol. 51, no. 11, pp. 18371841, 2006.

[6] H. Li and M. Fu, "Linear matrix inequality approach to robust $H_{\infty}$ filtering," IEEE Transactions on Signal Processing, vol. 45, no. 9, pp. 2338-2350, 1997.

[7] D. Yue and Q.-L. Han, "Network-based robust $H_{\infty}$ filtering for uncertain linear systems," IEEE Transactions on Signal Processing, vol. 54, no. 11, pp. 4293-4301, 2006.

[8] S. Xu, J. Lam, and X. Mao, "Delay-dependent $H_{\infty}$ control and filtering for uncertain Markovian jump systems with timevarying delays," IEEE Transactions on Circuits and Systems I, vol. 54, no. 9, pp. 2070-2077, 2007.

[9] C. E. de Souza, K. A. Barbosa, and A. Trofino Neto, "Robust $H_{\infty}$ filtering for discrete-time linear systems with uncertain timevarying parameters," IEEE Transactions on Signal Processing, vol. 54, no. 6 I, pp. 2110-2118, 2006.

[10] R. Yang, H. Gao, and P. Shi, "Delay-dependent $l_{2}-l_{\infty}$ filter design for stochastic time-delay systems," IET Control Theory \& Applications, vol. 5, no. 1, pp. 1-8, 2011.

[11] H. Zhang, A. S. Mehr, and Y. Shi, "Improved robust energy-topeak filtering for uncertain linear systems," Signal Processing, vol. 90, no. 9, pp. 2667-2675, 2010.

[12] M. S. Mahmoud, "Resilient $l_{2}-l_{\infty}$ filtering of polytopic systems with state delays," IET Control Theory \& Applications, vol. 1, no. 1, pp. 141-154, 2007.

[13] H. Zhang, Y. Shi, A. Saadat Mehr, and H. Huang, "Robust FIR equalization for time-varying communication channels with intermittent observations via an LMI approach," Signal Processing, vol. 91, no. 7, pp. 1651-1658, 2011.

[14] H. Gao and C. Wang, "Robust $l_{2}-l_{\infty}$ filtering for uncertain systems with multiple time-varying state delays," IEEE Transactions on Circuits and Systems I, vol. 50, no. 4, pp. 594-599, 2003.

[15] H. Zhang, Y. Shi, and A. Saadat Mehr, "Robust energy-to-peak filtering for networked systems with time-varying delays and randomly missing data," IET Control Theory \& Applications, vol. 4, no. 12, pp. 2921-2936, 2010.

[16] J. Qiu, G. Feng, and J. Yang, "New results on robust energyto-peak filtering for discrete-time switched polytopic linear systems with time-varying delay," IET Control Theory \& Applications, vol. 2, no. 9, pp. 795-806, 2008.

[17] S. He and F. Liu, "Robust peak-to-peak filtering for Markov jump systems," Signal Processing, vol. 90, no. 2, pp. 513-522, 2010.

[18] K. M. Grigoriadis and J. T. Watson Jr., "Reduced-order $H_{\infty}$ and $l_{2}-l_{\infty}$ filtering via linear matrix inequalities," IEEE Transactions on Aerospace and Electronic Systems, vol. 33, no. 4, pp. 1326-1338, 1997.

[19] R. M. Palhares and P. L. D. Peres, "Robust filtering with guaranteed energy-to-peak performance-an LMI approach," Automatica, vol. 36, no. 6, pp. 851-858, 2000.

[20] L. Zhang, P. Shi, E.-K. Boukas, and C. Wang, "Robust $l_{2}-l_{\infty}$ filtering for switched linear discrete time-delay systems with polytopic uncertainties," IET Control Theory \& Applications, vol. 1, no. 3, pp. 722-730, 2007.

[21] S.-H. Tsai, "A global exponential fuzzy observer design for timedelay Takagi-Sugeno uncertain discrete fuzzy bilinear systems with disturbance," IEEE Transactions on Fuzzy Systems, vol. 20, no. 6, pp. 1063-1075, 2012.

[22] H.-N. Wu, J.-W. Wang, and H.-X. Li, "Exponential stabilization for a class of nonlinear parabolic PDE systems via fuzzy control approach," IEEE Transactions on Fuzzy Systems, vol. 20, no. 2, pp. 318-329, 2012.

[23] X.-H. Chang, "Robust nonfragile $H_{\infty}$ filtering of fuzzy systems with linear fractional parametric uncertainties," IEEE Transactions on Fuzzy Systems, vol. 20, no. 6, pp. 1001-1011, 2012.

[24] J. Qiu, G. Feng, and H. Gao, "Observer-based piecewise affine output feedback controller synthesis of continuous-time T-S fuzzy affine dynamic systems using quantized measurements," IEEE Transactions on Fuzzy Systems, vol. 20, no. 6, pp. 10461062, 2012.

[25] J. Qiu, G. Feng, and H. Gao, "Static-output-feedback $H_{\infty}$ control of continuous-time T-S fuzzy affine systems via piecewise lyapunov functions," IEEE Transactions on Fuzzy Systems, vol. 21, no. 2, pp. 245-261, 2013.

[26] H. Zhang, Y. Shi, and M. X. Liu, " $H_{\infty}$ step tracking control for networked discrete-time nonlinear systems with integral and predictive actions," IEEE Transactions on Industrial Informatics, vol. 9, no. 1, pp. 337-345, 2013.

[27] H. Zhang, H. Zhong, and C. Dang, "Delay-dependent decentralized $H_{\infty}$ filtering for discrete-time nonlinear interconnected systems with time-varying delay based on the T-S fuzzy model," IEEE Transactions on Fuzzy Systems, vol. 20, no. 3, pp. 431-443, 2012.

[28] B.-S. Chen, W.-H. Chen, and W. Zhang, "Robust filter for nonlinear stochastic partial differential systems in sensor signal processing: Fuzzy approach," IEEE Transactions on Fuzzy Systems, vol. 20, no. 5, pp. 957-970, 2012.

[29] H. Li, H. Liu, H. Gao, and P. Shi, "Reliable fuzzy control for active suspension systems with actuator delay and fault," IEEE Transactions on Fuzzy Systems, vol. 20, no. 2, pp. 342-357, 2012.

[30] M. Chadli and T.-M. Guerra, "LMI solution for robust static output feedback control of discrete Takagi-Sugeno fuzzy models," IEEE Transactions on Fuzzy Systems, vol. 20, no. 6, pp. 11601165, 2012.

[31] X.-L. Zhu, B. Chen, D. Yue, and Y. Wang, "An improved input delay approach to stabilization of fuzzy systems under variable sampling," IEEE Transactions on Fuzzy Systems, vol. 20, no. 2, pp. 330-341, 2012.

[32] Y. Zhao and H. Gao, "Fuzzy-model-based control of an overhead crane with input delay and actuator saturation," IEEE Transactions on Fuzzy Systems, vol. 20, no. 1, pp. 181-186, 2012.

[33] H. Zhang and X. Xie, "Relaxed stability conditions for continuous-time TS fuzzy-control systems via augmented multi-indexed matrix approach," IEEE Transactions on Fuzzy Systems, vol. 19, no. 3, pp. 478-492, 2011.

[34] M. Chen, G. Feng, H. Ma, and G. Chen, "Delay-dependent $H_{\infty}$ filter design for discrete-time fuzzy systems with time-varying delays," IEEE Transactions on Fuzzy Systems, vol. 17, no. 3, pp. 604-616, 2009.

[35] T. M. Guerra, H. Kerkeni, J. Lauber, and L. Vermeiren, "An efficient lyapunov function for discrete T-S models: observer design," IEEE Transactions on Fuzzy Systems, vol. 20, no. 1, pp. 187-192, 2012. 
[36] H. Zhang, Y. Shi, and A. Saadat Mehr, "On $H_{\infty}$ filtering for discrete-time takagi-sugeno fuzzy systems," IEEE Transactions on Fuzzy Systems, vol. 20, no. 2, pp. 396-401, 2012.

[37] S. He and F. Liu, "Finite-time $H_{\infty}$ fuzzy control of nonlinear jump systems with time delays via dynamic observer-based state feedback," IEEE Transactions on Fuzzy Systems, vol. 20, no. 4, pp. 605-614, 2012.

[38] G. Feng, M. Chen, D. Sun, and T. Zhang, "Approaches to robust filtering design of discrete time fuzzy dynamic systems," IEEE Transactions on Fuzzy Systems, vol. 16, no. 2, pp. 331-340, 2008.

[39] J. An, G. Wen, C. Lin, and R. Li, "New results on a delayderivative-dependent fuzzy $H_{\infty}$ filter design for T-S fuzzy systems," IEEE Transactions on Fuzzy Systems, vol. 19, no. 4, pp. 770-779, 2011.

[40] H. Zhang, C. Dang, and C. Li, "Decentralized $H_{\infty}$ filter design for discrete-time interconnected fuzzy systems," IEEE Transactions on Fuzzy Systems, vol. 17, no. 6, pp. 1428-1440, 2009.

[41] H. H. Choi, "Robust stabilization of uncertain fuzzy-time-delay systems using sliding-mode-control approach," IEEE Transactions on Fuzzy Systems, vol. 18, no. 5, pp. 979-984, 2010.

[42] B.-S. Chen and C.-H. Wu, "Robust optimal reference-tracking design method for stochastic ynthetic biology systems: TS fuzzy approach," IEEE Transactions on Fuzzy Systems, vol. 18, no. 6, pp. 1144-1159, 2010.

[43] D. H. Lee, J. B. Park, and Y. H. Joo, "A new fuzzy lyapunov function for relaxed stability condition of continuous-time takagi-sugeno fuzzy systems," IEEE Transactions on Fuzzy Systems, vol. 19, no. 4, pp. 785-791, 2011.

[44] T. Takagi and M. Sugeno, "Fuzzy identification of systems and its applications to modeling and control," IEEE Transactions on Systems, Man and Cybernetics, vol. 15, no. 1, pp. 116-132, 1985.

[45] K. Tanaka and H. O. Wang, Fuzy Control Systems Design and Analysis: A Linear Matrix Inequality Approach, Wiley, New York, NY, USA, 2001.

[46] G. Feng, "A survey on analysis and design of model-based fuzzy control systems," IEEE Transactions on Fuzzy Systems, vol. 14, no. 5, pp. 676-697, 2006.

[47] H. Zhang, C. Dang, and J. Zhang, "Decentralized fuzzy $H_{\infty}$ filtering for nonlinear interconnected systems with multiple time delays," IEEE Transactions on Systems, Man, and Cybernetics Part B, vol. 40, no. 4, pp. 1197-1203, 2010.

[48] Z. Wang, F. Yang, D. W. C. Ho, and X. Liu, "Robust $H_{\infty}$ filtering for stochastic time-delay systems with missing measurements," IEEE Transactions on Signal Processing, vol. 54, no. 7, pp. 25792587, 2006.

[49] B. Chen, L. Yu, and W.-A. Zhang, "Robust Kalman filtering for uncertain state delay systems with random observation delays and missing measurements," IET Control Theory \& Applications, vol. 5, no. 17, pp. 1945-1954, 2011.

[50] Z. Wang, D. W. C. Ho, and X. Liu, "Variance-constrained filtering for uncertain stochastic systems with missing measurements," IEEE Transactions on Automatic Control, vol. 48, no. 7, pp. 1254-1258, 2003.

[51] H. Zhang, Y. Shi, and A. S. Mehr, "Robust weighted $H_{\infty}$ filtering for networked systems with intermittent measurements of multiple sensors," International Journal of Adaptive Control and Signal Processing, vol. 25, no. 4, pp. 313-330, 2011.

[52] H. Zhang, Q. Chen, H. Yan, and J. Liu, "Robust $H_{\infty}$ filtering for switched stochastic system with missing measurements," IEEE Transactions on Signal Processing, vol. 57, no. 9, pp. 3466-3474, 2009.
[53] H. Dong, Z. Wang, D. W. C. Ho, and H. Gao, "Varianceconstrained $H_{\infty}$ filtering for a class of nonlinear time-varying systems with multiple missing measurements: the finitehorizon case," IEEE Transactions on Signal Processing, vol. 58, no. 5, pp. 2534-2543, 2010.

[54] F. Yang and Y. Li, "Robust set-membership filtering for systems with missing measurement: a linear matrix inequality approach," IET Signal Processing, vol. 6, no. 4, pp. 341-347, 2012.

[55] S. M. K. Mohamed and S. Nahavandi, "Robust finite-horizon Kalman filtering for uncertain discrete-time systems," IEEE Transactions on Automatic Control, vol. 57, no. 6, pp. 1548-1552, 2012.

[56] B. Shen, Z. Wang, H. Shu, and G. Wei, "On nonlinear $H_{\infty}$ filtering for discrete-time stochastic systems with missing measurements," IEEE Transactions on Automatic Control, vol. 53, no. 9, pp. 2170-2180, 2008.

[57] F. Yang, Z. Wang, D. W. C. Ho, and M. Gani, "Robust $H_{\infty}$ control with missing measurements and time delays," IEEE Transactions on Automatic Control, vol. 52, no. 9, pp. 1666-1672, 2007.

[58] H. Zhang, Y. Shi, and B. Mu, "Optimal H1-based linear quadratic regulator tracking control for discrete-time TakagiSugeno fuzzy systems with preview actions," Journal of Dynamic Systems, Measurement, and Control, vol. 135, no. 4, Article ID 044501, 5 pages, 2013.

[59] H. Gao, Y. Zhao, J. Lam, and K. Chen, " $H_{\infty}$ fuzzy filtering of nonlinear systems with intermittent measurements," IEEE Transactions on Fuzzy Systems, vol. 17, no. 2, pp. 291-300, 2009. 


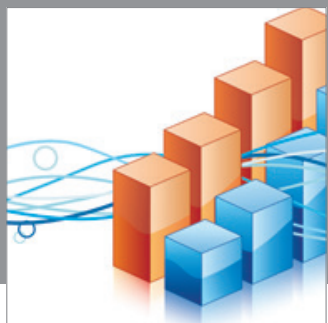

Advances in

Operations Research

mansans

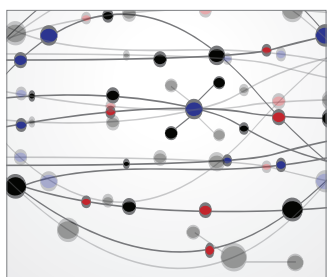

The Scientific World Journal
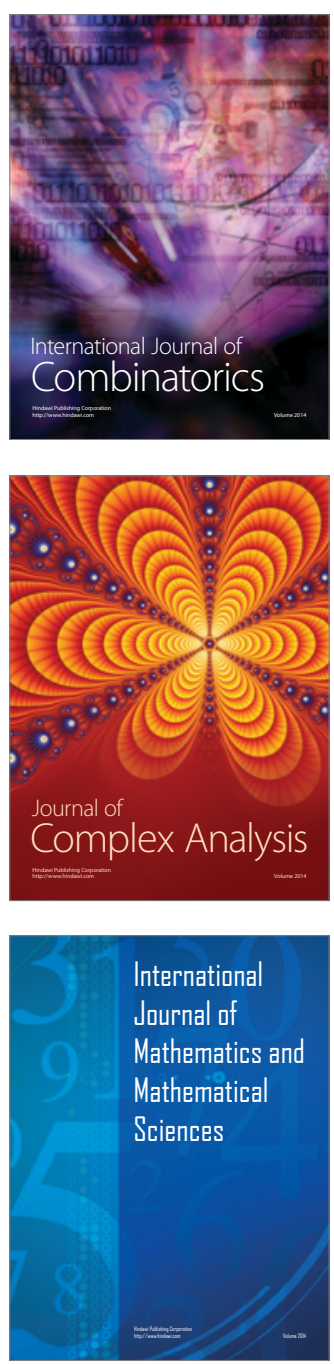
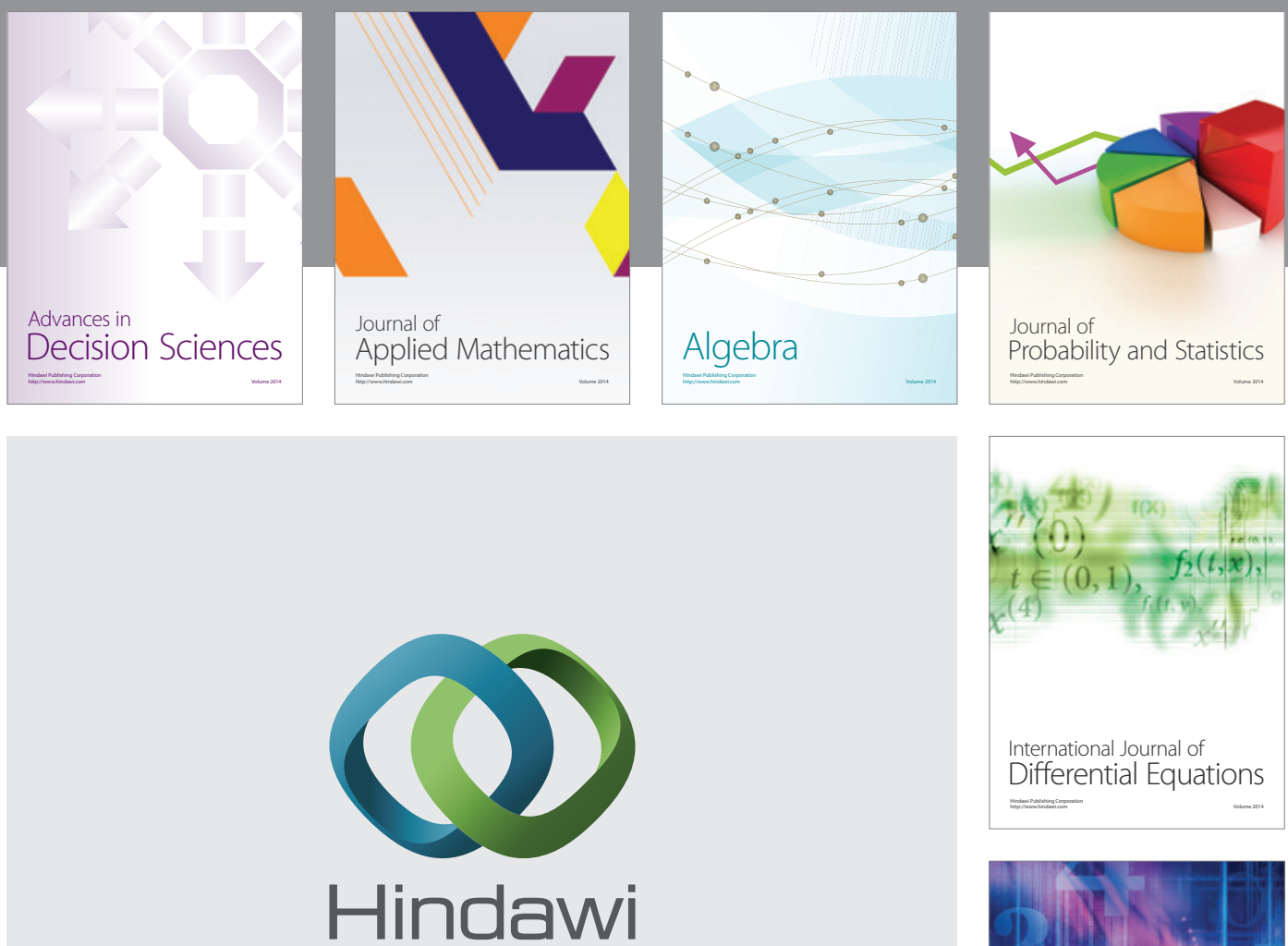

Submit your manuscripts at http://www.hindawi.com
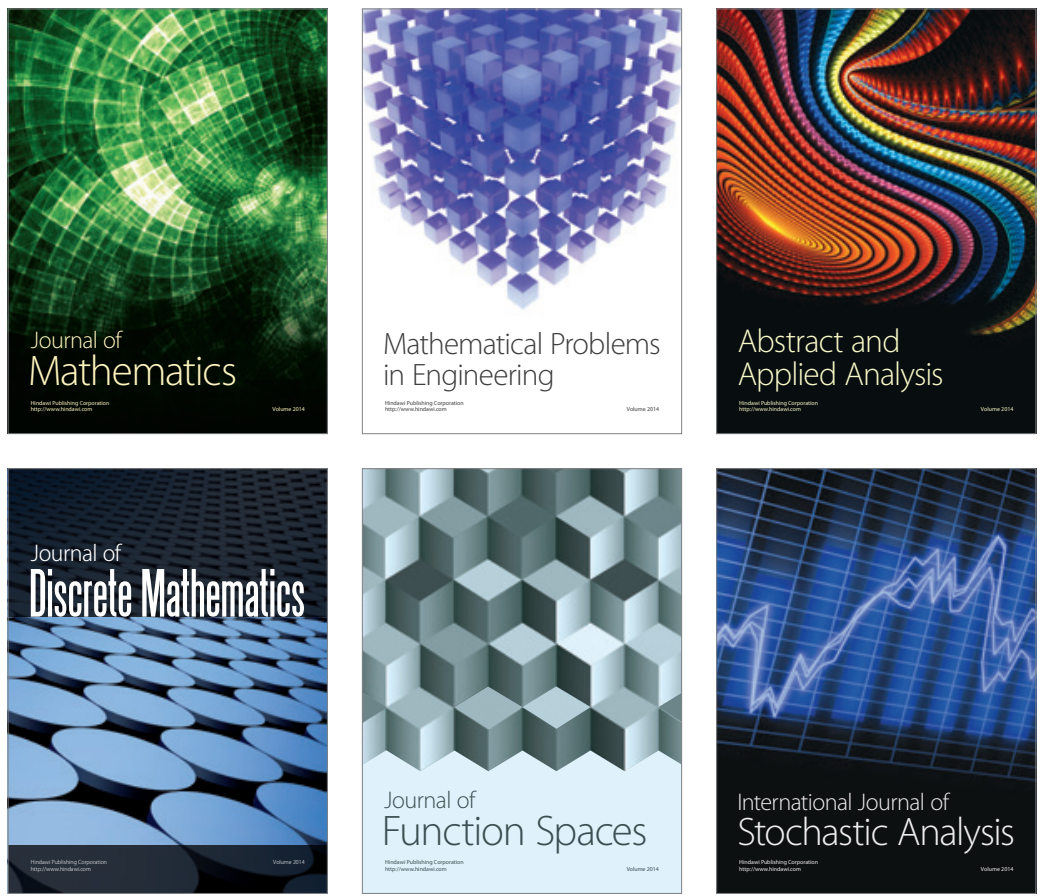

Journal of

Function Spaces

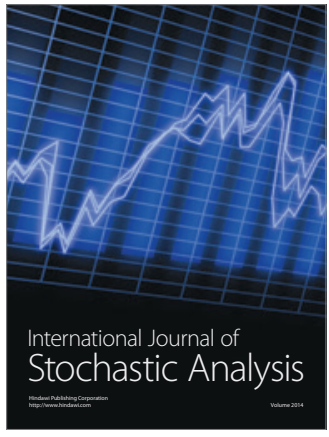

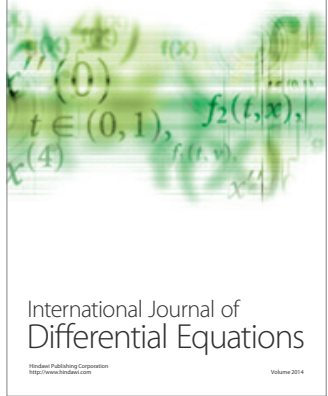
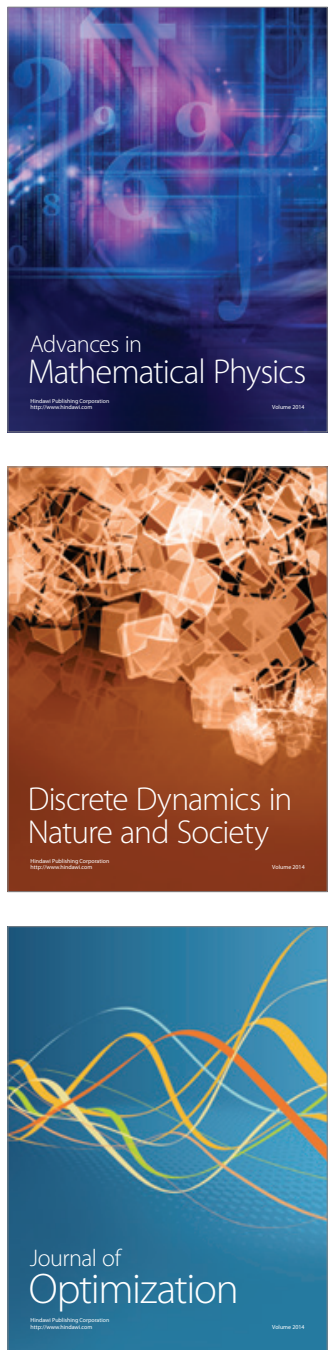